\title{
Research and Simulation of SCR Soft-Start Asynchronous Motor
}

\author{
Quan $\mathrm{Li}^{1, \mathrm{a}}$, Xin Wang ${ }^{2, \mathrm{~b}^{*}}$, Jun Zhou ${ }^{3}$ \\ ${ }^{1}$ College of Electrical Engineering, Shanghai University of Electric Power, Shanghai, China \\ ${ }^{2}$ Center of Electrical \& Electronic Technology, Shanghai Jiao Tong University, Shanghai, China \\ ${ }^{3}$ Maintenance Department of She'yang Power Supply Company of State Grid Jiangsu Electric \\ Power Company, China \\ aliquan9388@163.com, bwangxin26@sjtu.edu.cn \\ *corresponding author: wangxin26@sjtu.edu.cn
}

\begin{abstract}
Keywords: three-phase asynchronous motor; SCR; soft-start; simulation
Abstract. Direct starting method of asynchronous motor does a lot harm such as current surge and mechanical shock, etc. In order to reduce the harmful influence of direct starting method and improve the starting performance of asynchronous motor, a scheme of asynchronous motor soft starting system based on antiparallel SCR is designed in this paper. Firstly, it analyzes the starting performance of asynchronous motor. Secondly, based on the performance results above, the scheme of three-phase asynchronous motor soft starting system is established. It regulates stator voltage of the motor by SCR regulating system, transformers the synchronous phase voltage to wide pulse wave by trigger module and obtains thyristor gate signal by integrator, so as to achieve the soft-start of asynchronous motor. Finally, by comparing and analyzing the simulation results of direct starting system and soft starting system, it shows that the feasibility and advantages of the SCR type's asynchronous motor soft starting system.
\end{abstract}

\section{Introduction}

In modern society, asynchronous motor plays a crucial role in the production of modern industry especially in the industry of electric driving. However, direct starting of the asynchronous motor would cause a current surge in the stator while it being activated in case of it has a higher normal rated power. This current surge will not only be harmful to the motor's working but also cause strong interference to electric power grid and, even cause power system split when the problem gets serious. This influence will reduce the service life of the electrical and mechanical equipment in the long run .

With the booming development of power electronics industrialization and the innovation of production technology, many kinds of motor soft starting device appeared, such as CST soft-start [1], magnetically controlled soft-start [2], thermal resistor soft-start [3], etc. Besides, the CST soft starting system is usually used for conveying systems, and the magnetically controlled soft-start and thermal resistor soft-start are more suitable for high voltage grid. At the same time, a sort of soft starting system with SCR type has attracted widespread attention because of its wide range applicability. This scheme not only solves the problem of current impulse and torque impulse in the direct starting process of the motor, but also can be used to different application conditions by setting its working state, which is very flexible and adaptable [4].

This paper has presented that SCR type soft-start scheme. Firstly, it analyzes the theory for the start performance of three-phase asynchronous motor. Secondly, it applies SCR module, trigger module and control module to achieve the soft starting process according to the theoretical research, and established the corresponding simulation model. Finally, it analyses the result of simulation of the soft-start motor system and compares it with that of direct starting process. The simulation result shows that the soft starting by SCR type will produce smaller stator current and no torque impulse when the motor starts. 


\section{Theoretical research on asynchronous motor starting}

In the theory about three-phase asynchronous motor, the rotor winding can be converted to the stator winding in general, and the excitation branch is placed in the input side. So the single-phase of asynchronous motor can be simplified as a $\Gamma$ type equivalent circuit [5]. As shown in Fig. 1.

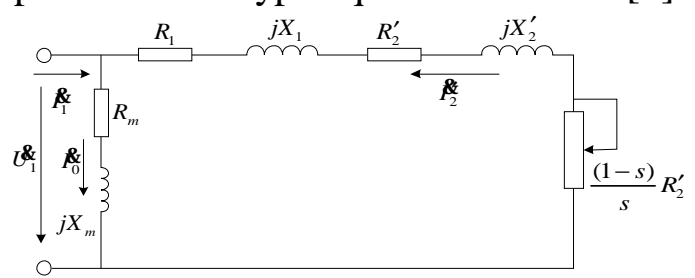

Fig. $1 \Gamma$ type equivalent circuit

Based on such equivalent circuit, the rotor current can be calculated:

$$
I_{2}^{\prime}=\frac{U_{1}}{\sqrt{\left(R_{1}+\frac{R_{2}^{\prime}}{s}\right)^{2}+\left(X_{1}+X_{2}^{\prime}\right)^{2}}} \text {. }
$$

For asynchronous motor, the stator and rotor leakage impedance is comparatively small. So when it starts, the starting current is mainly composed of stator and rotor starting current. What's more, the excitation current can be neglected.

$$
T=\frac{3 I_{2}^{\prime} \frac{R_{2}^{\prime}}{s}}{\frac{2 \pi n_{1}}{60}}=\frac{3 I_{2}^{\prime 2} \frac{R_{2}^{\prime}}{s}}{\frac{2 \pi f_{1}}{p}}=\frac{3 p U_{1}^{2} \frac{R_{2}^{\prime}}{s}}{2 \pi f_{1}\left[\left(R_{1}+\frac{R_{2}^{\prime}}{s}\right)^{2}+\left(X_{1}+X_{2}^{\prime}\right)^{2}\right]} .
$$

The Eq. 2 is that parameter expression of the mechanical performance of asynchronous motor [2]. By the Eq. 1 and Eq. 2 which are expressions of rotor current and electromagnetic torque. In the case of direct starting, $\mathrm{U}_{1}$ is in a high level in the starting moment which may cause the impulse of current and torque. On the other hand, the theory of soft starting with SCR type is to achieve the purpose of changing the mechanical characteristics and controlling the starting torque and starting current of the motor by adjusting the stator voltage in the starting process[6].

\section{SCR soft starting system}

The SCR soft starting system is composed of the SCR module, the trigger module and the control module $[7,8]$.

SCR module. It is mainly composed of two antiparallel thyristor, as shown in Fig. 2. That three of such module is needed to constitute the main circuit of three-phase AC voltage control circuit.

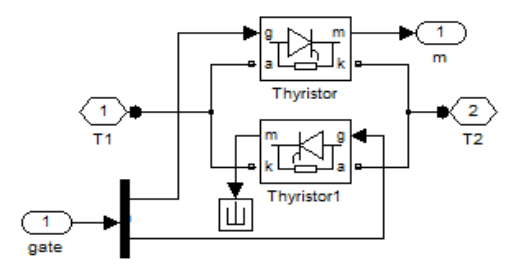

Fig. 2 SCR module

Trigger module. AC voltage regulator control circuit, which includes square wave forming part, sawtooth wave forming part and phase-shifting control part. 


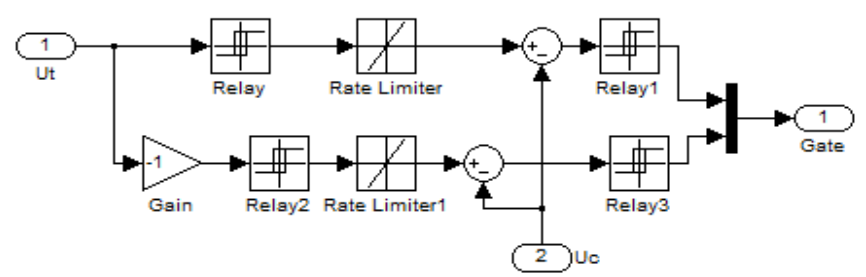

Fig. 3 Trigger module

As shown in Fig. 3, Ut is the synchronous phase voltage, which became a part of square wave by the relay module. The square wave shares the same width of positive half cycle of the synchronous phase, and became a part of sawtooth wave after the Rate Limiter. The trigger module achieves the purpose of changing zero crossing point of the saw tooth wave voltage by subtracting $U_{c}$ from sawtooth wave, which is shaped into 180 degree wide pulse finally [4].

Control module. It is shown in follow figure Fig. 4.

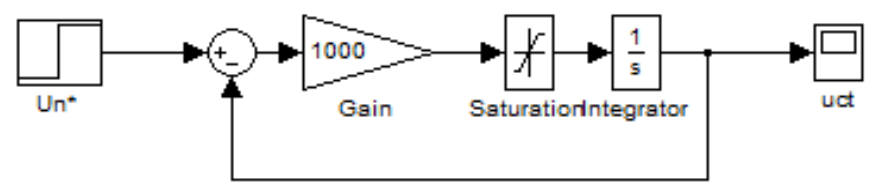

Fig. 4 Control module

Phase-shifting control voltage Uc which is a ramp signalis formed by control module. These module constitute the simulation model of three-phase asynchronous motor with SCR soft starting system, as shown in Fig. 5.

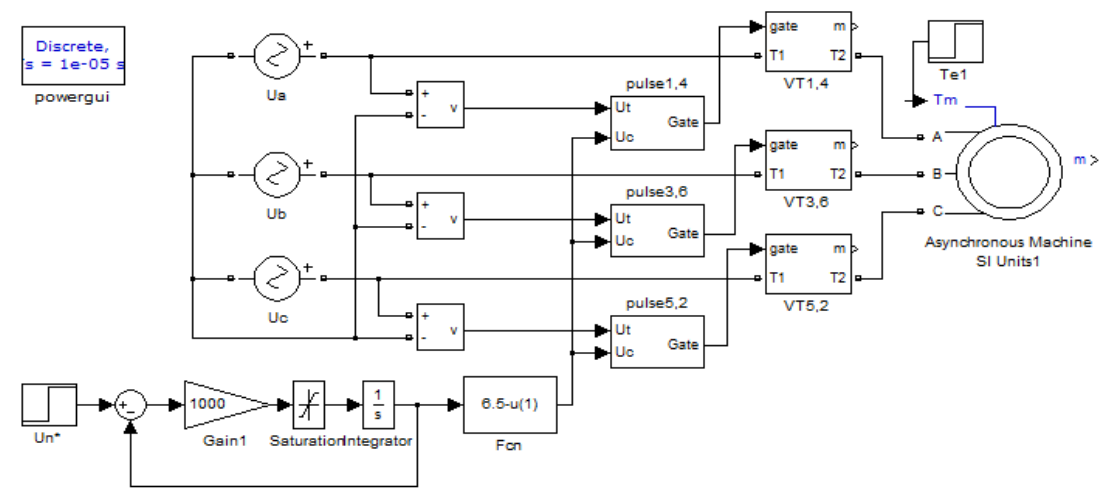

Fig. 5 Simulation model

\section{Simulation results and analysis}

The no-load starting process of the motor is simulated by that simulation model, whose results is compared with direct starting of the motor.
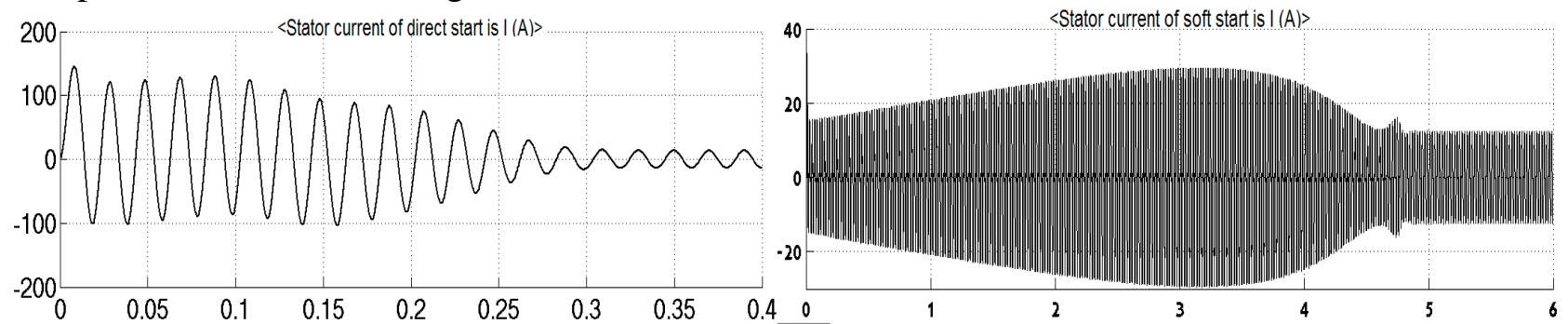

Fig. 6 Stator current of starting process 

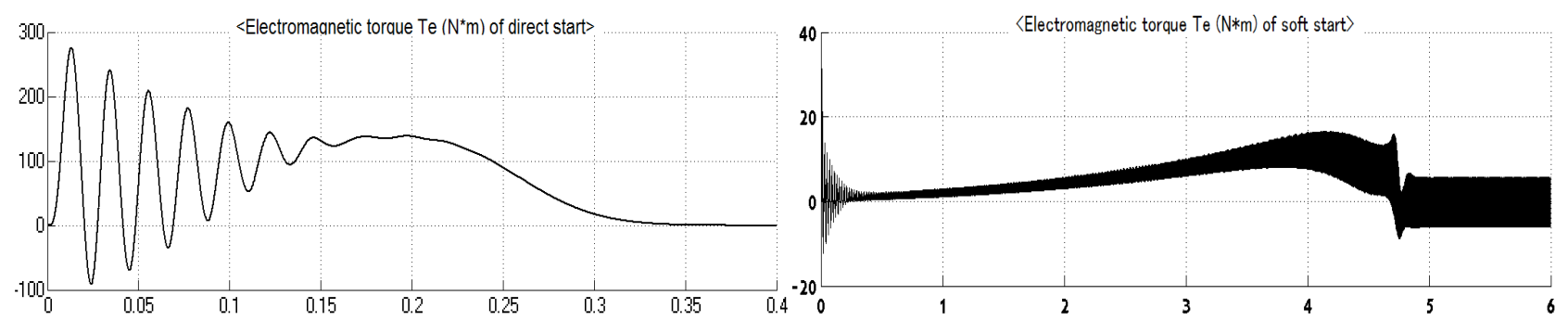

Fig. 7 Electromagnetic torque of starting process

As shown in Fig. 6 and 7, the time (approx 0.3s) used for the direct starting process is relatively short. When the motor starts up, the stator current is over 100A, which almost reached five times of the rated current. What's more, when the motor starts ( 0 seconds), the electromagnetic torque is about $300 \mathrm{~N} \bullet \mathrm{m}$, which is a relatively large torque. It is dangerous to the motor with low mechanical strength and may hurt the motor and mechanics. In the other hand, the starting current of SCR soft starting motor is smooth and the maximum value is not more than $30 \mathrm{~A}$, which can eliminate the adverse effects caused by the impact current. The current changes in the whole soft starting process are relatively stable. Although electromagnetic torque at the start of the starting is not large, but with the process of adjusting the voltage continues, the voltage of stator is also rising, the speed is gradually increased, finally reach the rated speed.

\section{Conclusions}

This paper designs a soft starting scheme of asynchronous motor by SCR type, which is achieved in simulation. From the processes of theoretical research, model building and analysis of the results, these conclusions can be obtained as the following.

(1) The simulation results prove that the direct starting has the problem of the current surge and the mechanical shock, which is inevitable in the direct starting process.

(2) This SCR type's soft-start scheme can effectively decrease the stator current and electromagnetic torque during starting process which is smooth and safe to the motor system. The starting performance of three-phase asynchronous motor can be improved by adopting this method.

(3) It is worth to pay attention that the soft starting technology is a kind of power electronic technology which contains a number of power electronic devices, so it will produce a certain harmonic in the system.

\section{Acknowledgements}

This work is supported by the Key Project of NSFC (No. 61533012), the Shanghai Natural Science Foundation (14ZR1421800), the State Key Laboratory of Synthetical Automation for Process Industries.

\section{References}

[1] Y. Lang, L. Wang, A plication of CST soft starting devices in transfer system, North China Electric Power. 07 (2002) 50-51.

[2] X. Pan, Y. Qiao, Study on several kinds of HV voltage-reducing soft starting method, Science \& Technology Information. 03 (2012) 128-129.

[3] Y. Zhang, Application of thermal variable resistance soft starting unit during fan starting, Compressor Blower \& Fan Technology. 01 (2005) 59-60.

[4] X. Chang,V. Cocquempot, S. Zhao, C. Christophe, New model of three phase induction motor and its simulation and experiment, Proceedings of the Csee. 23 (2003) 140-145. 
[5] L. Xie, Z. Wang, W. Wu, Research on the start up characteristic of 3-phase Squirrel cage asynchronous motors, Process Automation Instrumentation. 09 (2011) 12-17.

[6] L. Fan, L. Zhang, Simulation of fuzzy soft start of asynchronous motor, Proceedings of the Chinese Society of Universities for Electric Power System and its Automation. 23 (2011) 123-126.

[7] Mehdi Arehpanahi, Jafar Mili Monfared, Karim Abbaszadeh. Sensorless fuzzy logic soft start of induction motor with load detection, Proceedings of the Eighth International Conference on Electrical Machines and Systems. 03 (2005).

[8] Y. Zhu, Research on intelligent soft starting method for three-phase asynchronous motor based on MATLAB, Advanced Materials Research. 1363 (2011) 828-831 